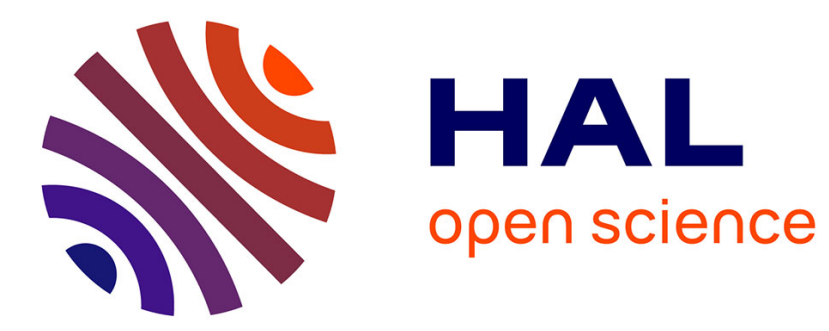

\title{
Matching of local expansions in the theory of non-linear vibrations
}

Yuri Mikhlin

\section{To cite this version:}

Yuri Mikhlin. Matching of local expansions in the theory of non-linear vibrations. Journal of Sound and Vibration, 1995, 182 (4), pp.577-588. 10.1006/jsvi.1995.0218 . hal-01347418

\section{HAL Id: hal-01347418 \\ https://hal.science/hal-01347418}

Submitted on 21 Jul 2016

HAL is a multi-disciplinary open access archive for the deposit and dissemination of scientific research documents, whether they are published or not. The documents may come from teaching and research institutions in France or abroad, or from public or private research centers.
L'archive ouverte pluridisciplinaire HAL, est destinée au dépôt et à la diffusion de documents scientifiques de niveau recherche, publiés ou non, émanant des établissements d'enseignement et de recherche français ou étrangers, des laboratoires publics ou privés. 


\title{
MATCHING OF LOCAL EXPANSIONS IN THE THEORY OF NON-LINEAR VIBRATIONS
}

\author{
Yu. V. MikHLIN \\ Dnepropetrovsk State University, 320050 Dnepropetrovsk, Ukraine
}

\begin{abstract}
Normal vibrations in non-linear systems are a generalization of normal (principal) vibrations of linear systems [1-3]. In this case all position co-ordinates can be defined well from any one of them. R. M. Rosenberg is credited with being the first to introduce broad classes of conservative systems allowing normal vibrations with rectilinear trajectories in a configurational space. In systems of a more general type, trajectories of normal vibrations are curvilinear. Assume that in a conservative system the potential energy is a positively definite polynomial in the co-ordinates. At small amplitudes a linear system is to be selected as the initial one, while at large amplitudes a homogeneous non-linear system allows normal vibrations with rectilinear trajectories. In the vicinity of a linear system, trajectories of normal vibrations can be determined as power series in the amplitude; while in the vicinity of a homogeneous non-linear system, they can be determined as power series in the inverse amplitude. In order to join together local expansions and to investigate the behavior of normal vibration trajectories at arbitrary amplitude values, fractional rational diagonal Padé approximants are used. Necessary conditions for the convergence of a succession of Padé approximants have been obtained, and that allows one to establish relations between quasi-linear and essentially non-linear expansions: that is, to decide which of them correspond to the same solution and which to different ones. Additional modes of vibrations exist only in a non-linear systems; as the amplitude decreases, they vanish at a certain limiting point.
\end{abstract}

\section{INTRODUCTION: NORMAL VIBRATIONS OF NON-LINEAR SYSTEMS}

Normal vibrations in the non-linear case are a generalization of normal (principal) vibrations of linear systems. In a normal mode a finite-dimensional system behaves like a conservative one, having a single degree of freedom. In this case all position co-ordinates can be defined well from any one of them by

$$
x_{i}=p_{i}(x), \quad x \equiv x_{1}, \quad i=2,3, \ldots, n,
$$

$p_{i}(x)$ being analytical functions.

Rosenberg [1,2] is credited as being the first to introduce broad classes of essentially non-linear (not quasi-linear) conservative systems allowing normal vibrations with rectilinear trajectories in a configurational space:

$$
x_{i}=k_{i} x_{1}, \quad i=2,3, \ldots, n .
$$

For instance, homogeneous systems the potential of which is an even homogeneous function of the variables belong to such a class. It is interesting to note that the number of modes of normal vibrations in the non-linear case can exceed the number of degrees of freedom. This remarkable property has no analogy in the linear (non-degenerate) case. 
In systems of a more general type, trajectories of normal vibrations are curvilinear. Liapunov [4] showed that solutions of this kind exist in non-linear finite-dimensional systems with an analytical first integral which are close to generating linear systems. New results concerning normal vibrations with curvilinear trajectories in essentially non-linear cases have been reported in references [3,5].

Consider a conservative system

$$
m_{i} \ddot{x}_{i}+\Pi_{x_{i}}=0, \quad \dot{x}_{i}=\mathrm{d} x_{i} / \mathrm{d} t, \quad \Pi_{z}=\partial \Pi / \partial z, \quad i=1,2, \ldots, n,
$$

$\Pi=\Pi(x)$ being the potential energy, assumed to be a positive definite function, and $x=\left(x_{1}, x_{2}, \ldots, x_{n}\right)^{\mathrm{T}}$. The power series expansion for $\Pi(x)$ begins with terms having a power of at least two. Without reducing the degree of generalization, assume that $m_{i}=1$, since this can be always ensured by dilatation of co-ordinates.

The energy integral for system (3) is

$$
\frac{1}{2} \sum_{k=1}^{n} \dot{x}_{k}^{2}+\Pi\left(x_{1}, x_{2}, \ldots, x_{n}\right)=h
$$

$h$ being the system energy. Assume that within the configuration space, bounded by a closed maximum equipotential surface $\Pi=h$, the only equilibrium position is $x_{i}=0$ $(i=1,2, \ldots, n)$.

Introduce a new independent variable $x$ instead of $t$ here. Since

$$
\frac{\mathrm{d}}{\mathrm{d} t}=\frac{\mathrm{d}}{\mathrm{d} x} \dot{x} \quad \text { and } \quad \frac{\mathrm{d}^{2}}{\mathrm{~d} t^{2}}=\frac{\mathrm{d}^{2}}{\mathrm{~d} x^{2}} \dot{x}^{2}+\frac{\mathrm{d}}{\mathrm{d} x} \ddot{x},
$$

and it follows from the integral of energy (4) that

$$
\dot{x}^{2}=2(h-\Pi) /\left\{1+\sum^{n}\left(x_{k}^{\prime 2}\right)\right\}
$$

the sought-for equations defining the trajectories in the configurational space are obtained to replace equations (3):

$$
2 x_{i}^{\prime \prime} \frac{h-\Pi}{1+\sum_{k=2}^{n}\left(x_{k}^{\prime}\right)^{2}}+x_{i}^{\prime}\left(-\Pi_{x}\right)=-\Pi_{x_{i}}, \quad i=2,3, \ldots, n, \quad x=x_{i} .
$$

These could be obtained, as well as Euler equations for the variational principle in the Jacobi form. Equations (5) are non-linear and non-autonomous, and have movable singular points: they are no simpler in the linear case. Nevertheless, these relatioships are suitable for the determination of nearly rectilinear trajectories of normal vibrations.

An analytical extension of the trajectories on a maximum isoenergy surface $\Pi=h$ is possible if the boundary conditions (i.e., conditions of orthogonality of a trajectory to the surface) are satisfied [2, 3]:

$$
x_{i}^{\prime}\left[-\Pi_{x}\left(X, x_{2}(X), \ldots, x_{n}(X)\right)\right]=-\Pi_{x_{i}}\left(X, x_{2}(X), \ldots, x_{n}(X)\right) .
$$

Here $\left(X, x_{2}(X), \ldots, x_{n}(X)\right)$ are the trajectory return points lying on the $\Pi=h$ surface where all velocities are equal to zero. If a trajectory $x_{i}(x)$ is defined, the law of motion with respect to time can be found by using

$$
\ddot{x}+\Pi_{x}\left(x, x_{2}(x), \ldots, x_{n}(x)\right)=0,
$$

for which a periodic solution $x(t)$ is obtained by inversion of the integral. 
Mikhlin et al. $[3,5]$ developed nearly rectilinear trajectories of normal vibrations in the form of power series.

Assume, for example, that $\Pi=\Pi_{0}+\epsilon \Pi_{1}$, where $\epsilon$ is a small parameter, and that the undisturbed system permits solution (2). The co-ordinate axes can be rotated so that a generating solution will be defined by $x_{i}=0(i=2,3, \ldots, n)$.

The solution can be found in the form of a convergent series:

$$
x_{i}=\sum_{k=1}^{\infty} \sum_{j=0}^{\infty} \epsilon^{k} a_{i j}^{(k)} x^{j}
$$

Specifically, if the generating system is homogeneous, i.e., $\Pi_{0}$ is an homogeneous even function of the power $r+1$ in all variables $(r=1,3,5, \ldots)$, the coefficients $a_{i j}^{(k)}$ will be well defined from equation (5) and the boundary conditions (6) when the following determinants are non zero: i.e.,

$$
k_{p}=\left|q_{i j}\right| \neq 0,
$$

where

$$
q_{i j}=\delta_{i}^{i}\left[p(p-1) 2 \Pi_{0}(1,0, \ldots, 0)+p \Pi_{0 x}(1,0, \ldots, 0)-\Pi_{0 x_{i} x_{i}}(1,0, \ldots, 0)\right],
$$

and $\delta_{i}^{j}$ are the Kronecker symbols, $p=0,1,2,3, \ldots$.

At $r=1$ conditions (8) become $\omega_{i} \neq \omega_{j}$, which for Liapunov systems mean that the case of inner resonance in the generating linear system is not considered.

\section{TWO-POINT PADÉ APPROXIMANTS FOR NORMAL VIBRATIONS}

Now consider a problem of normal vibrational behavior in certain non-linear systems when the amplitude (or energy) of the vibrations is varied from zero to an extremely large value.

Assume that in a system

$$
\ddot{z}_{i}+\Pi_{z_{i}}\left(z_{1}, z_{2}, \ldots, z_{n}\right)=0
$$

the potential energy $\Pi\left(z_{1}, z_{2}, \ldots, z_{n}\right)$ is a positive definite polynomial of $z_{1}, \ldots, z_{n}$ having a minimum power of two and a maximum power of $2 \mathrm{~m}$. On choosing a co-ordinate, say $z_{1}$, substitute $z_{i}=c x_{i}$, where $c=z_{1}(0)$. Obviously, $x_{1}(0)=1$. Furthermore, without loss of generality, assume that $\dot{x}_{1}(0)=0$. Equation (9) can be rewritten as

$$
\ddot{x}_{i}+V_{x_{i}}\left(c, x_{1}, x_{2}, \ldots, x_{n}\right)=0,
$$

where

$$
V=\sum_{k=0}^{2 m-2} c^{k} V^{(k+z)}\left(x_{1}, x_{2}, \ldots, x_{n}\right)
$$

and $V^{(r+1)}$ contains terms of power $(r+1)$ of the variables in the potential

$$
V\left(c, x_{1}, x_{2}, \ldots, x_{n}\right) \equiv \Pi\left(z_{1}\left(x_{1}\right), z_{2}\left(x_{2}\right), \ldots, z_{n}\left(x_{n}\right)\right) .
$$

The energy integral here is

$$
\frac{1}{2} c^{2}\left(\dot{x}_{1}^{2}+\sum_{i=z}^{n} \dot{x}_{i}^{2}\right)+V\left(c, x_{1}, x_{2}, \ldots, x_{n}\right)=h,
$$

$h$ being the system energy. It is assumed below that the amplitude of vibration $c=z_{1}(0)$ is the independent parameter, while the energy is defined by equation (11). Therefore it 
is expedient to represent the energy $h$ as a sum of summands corresponding to homogeneous components of the potential energy $V$ :

$$
h=\sum_{k=2}^{2 m} c^{k} h_{k}
$$

In order to determine trajectories of the normal vibrations of the initial system, equations (5) are used together with boundary conditions (6).

At small amplitudes an homogeneous linear system with a potential energy $V^{(2)}$ is selected as the initial one while, at large amplitude, an homogeneous non-linear system with a potential energy $V^{(2 m)}$ is selected. Both linear and non-linear homogeneous systems allow normal vibrations of the $x_{i}=k_{i} x_{1}$ type, where the constants $k_{i}$ are determined from the algebraic equations

$$
k_{i} V_{x_{1}}^{(r)}\left(1, k_{2}, \ldots, k_{n}\right)=V_{x_{i}}^{(r)}\left(1, k_{2}, \ldots, k_{n}\right) .
$$

(In the non-linear case the number of vibrations of this type can be greater than the number of degrees of freedom.)

In the vicinity of a linear system at small values of $c$ trajectories of normal vibrations can be determined as power series of $x$ and $c$ (upon assuming that $x_{1} \equiv x$ ),

$$
x_{i}^{(1)}=\sum_{j=0}^{\infty} \alpha_{j}^{(i)}(x) c^{j} \equiv \sum_{j=0}^{\infty} \sum_{l=0}^{\infty} \alpha_{j l}^{(i)} x^{l} c^{j}, \quad i=2,3, \ldots, n,
$$

while in the vicinity of a homogeneous non-linear system (at large values of $c$ ), as power series of $x$ and $c^{-1}$.

$$
x_{i}^{(2)}=\sum_{j=0}^{\infty} \beta_{j}^{(i)}(x) c^{-j} \equiv \sum_{j=0}^{\infty} \sum_{l=0}^{\infty} \beta_{j l}^{(i)} x^{l} c^{-j}, \quad i=2,3, \ldots, n .
$$

The construction of series (13) and (14) is described above.

Note that functions $\alpha_{j}(x)$ and $\beta_{j}(x)$ can be obtained in quadrature formulas as well, since the variational equations for normal vibrations of homogeneous systems are reduced to hypergeometric ones.

The amplitude values $x=1, x_{i}^{(1)}(1)$ and $x_{i}^{(2)}(1)$ (at $\dot{x}=\dot{x}_{i}=0$ ) define the normal vibrations mode completely. Therefore, for the sake of simplicity, only expansions of $\rho_{i}^{(1)}=x_{i}^{(1)}(1)$ and $\rho_{i}^{(2)}=x_{i}(1)$ in terms of powers of $c$ will be discussed below, which are obtained from equations (13) and (14) at $x=1$ (denoted as $\alpha_{j}^{(i)}=a_{j}^{(i)}(1)$ and $\beta_{j}^{(i)}=\beta_{j}^{(i)}(1)$ ):

$$
\rho_{i}^{(1)}=\sum_{j=0}^{\infty} \alpha_{j}^{(i)} c^{j}, \quad \rho_{i}^{(2)}=\sum_{j=0}^{\infty} \beta_{j}^{(i)} c^{-j}
$$

In order to join together local expansions (15) and to investigate the behavior of normal vibration trajectories at arbitrary values of $c$, fractional rational diagonal Padé approximants are used [5-7]:

$$
P_{s}^{(i)}=\sum_{j=0}^{s} a_{j}^{(i)} c^{j} / \sum_{j=0}^{s} b_{j}^{(i)} c^{j}, \quad s=1,2,3, \ldots, \quad i=2,3, \ldots, n .
$$

Along with the Padé approximants in terms of positive powers of $c$ one can write those in terms of powers of $c^{-1}$ :

$$
P_{s}^{(i)}=\sum_{j=0}^{s} a_{j}^{(i)} c^{j-s} / \sum_{j=0}^{s} b_{j}^{(i)} c^{j-s}, \quad s=1,2,3, \ldots, \quad i=2,3, \ldots, n .
$$


Compare the expressions (16) and (17) with expansions (15):

$$
\begin{gathered}
\sum_{j=0}^{\infty} \alpha_{j}^{(i)} c^{j} \simeq \sum_{j=0}^{s} a_{j}^{(i)} c^{j} / \sum_{j=0}^{s} b_{j}^{(i)} c^{j}, \\
\sum_{j=0}^{s} a_{j}^{(i)} c^{j-s} / \sum_{j=0}^{s} b_{j}^{(i)} c^{j-s} \simeq \sum_{j=0}^{\infty} \beta_{j}^{(i)} c^{-j},
\end{gathered}
$$

or

$$
\begin{aligned}
\left(\sum_{j=0}^{\infty} \alpha_{j}^{(i)} c^{j}\right)\left(\sum_{j=0}^{s} b_{j}^{(i)} c^{j}\right) & \simeq \sum_{j=0}^{s} a_{j}^{(i)} c^{j}, \\
\left(\sum_{j=0}^{\infty} \beta_{j}^{(i)} c^{-j}\right)\left(\sum_{j=0}^{s} b_{j}^{(i)} c^{j-s}\right) & \simeq \sum_{j=0}^{s} a_{j}^{(i)} c^{j-s} .
\end{aligned}
$$

By preserving in equations (18) only the terms with an order of $c^{r}(-s \leqslant r \leqslant s)$ and equating the coefficients of equal powers of $c, n-1$ systems of $2(s+1)$ linear algebraic equations will be obtained for determination of $a_{j}^{(i)}, b_{j}^{(i)}, j=0,1,2, \ldots$.

The determinants of these systems take the form of

$$
\begin{aligned}
& s+1 \quad s+1 \\
& s+1\left\{\begin{array}{rrrrrrrrrrr}
\overbrace{-1}^{1} & 0 & \ldots & 0 & 0 & \alpha_{0} & 0 & \ldots & 0 & 0 \\
0 & -1 & \ldots & 0 & 0 & \alpha_{1} & \alpha_{0} & \ldots & 0 & 0 \\
\ldots & \ldots & \ldots & & \ldots & \ldots & & \ldots & \\
0 & 0 & \cdots & -1 & 0 & \alpha_{s-1} & \alpha_{s-2} & \ldots & \alpha_{0} & 0 \\
0 & 0 & \ldots & 0 & -1 & \alpha_{s} & \alpha_{s-1} & \ldots & \alpha_{1} & \alpha_{0}
\end{array}\right. \\
& \Delta_{s}^{(i)}=\quad \begin{array}{rrrrrrrrrrr}
0 & 0 & \cdots & 0 & -1 & \alpha_{s} & \alpha_{s-1} & \cdots & \alpha_{1} & \alpha_{0} \\
-1 & 0 & \cdots & 0 & 0 & \beta_{0} & \beta_{1} & \cdots & \beta_{s-1} & \beta_{s}
\end{array} \\
& s+1\left\{\begin{array}{rrrrrrrrrr}
-1 & & & & & & & & & \\
0 & -1 & \cdots & 0 & 0 & 0 & \beta_{0} & \ldots & \beta_{s-2} & \beta_{s-1} \\
\ldots & & \ldots & \ldots & & & \ldots & \ldots & \ldots & \ldots \\
0 & 0 & \cdots & -1 & 0 & 0 & 0 & \ldots & \beta_{0} & \beta_{1} \\
0 & 0 & \cdots & 0 & -1 & 0 & 0 & \ldots & 0 & \beta_{0}
\end{array}\right. \\
& =\left|\begin{array}{ll}
I_{s+1}^{(-)} & D_{(\alpha) s+1} \\
I_{s+1}^{(-)} & D_{(\beta) s+1}^{\mathrm{T}}
\end{array}\right|,
\end{aligned}
$$

where

$$
I_{s+1}^{(-1)}=\left|\begin{array}{rrrr}
-1 & 0 & \cdots & 0 \\
0 & -1 & \cdots & 0 \\
\ldots & & \cdots & \cdots \\
0 & 0 & \cdots & -1
\end{array}\right|, \quad D_{(\gamma) s+1}=\left|\begin{array}{cccc}
\gamma_{0} & 0 & \ldots & 0 \\
\gamma_{1} & \gamma_{0} & \ldots & 0 \\
\ldots & \ldots & \ldots \\
\gamma_{s} & \gamma_{s-1} & \ldots & \gamma_{0}
\end{array}\right|,
$$

and $s+1$ is the order of the determinant.

Since the determinants are generally not equal to zero, the systems of algebraic equations have a single exact solution, $a_{j}^{(i)}=b_{j}^{(i)}=0$.

Select a Padé approximant corresponding to the preserved terms in equation (15) having non-zero coefficients $a_{j}^{(1)}$ and $b_{j}^{(1)}$. Assume that $b_{0}^{(i)} \neq 0$, for otherwise at $c \rightarrow 0, x_{i}^{(1)} \rightarrow \infty$. Without loss of generality, it can also be assumed that $b_{0}^{(i)}=1$. Now the systems of algebraic equations for determination of $a_{j}^{(i)}$ and $b_{j}^{(i)}$ become overdetermined. All unknown coefficients $a_{0}^{(i)}, \ldots, a_{n}^{(i)}, b_{1}^{(i)}, \ldots, b_{n}^{(i)}, i=2,3, \ldots, n$, are determined from $2 s+1$ 
equations, while the "error" of this approximate solution can be obtained by substitution of all coefficients in the remaining equation. Obviously, the "error" is determined by the value of $\Delta_{s}^{(i)}$, since at $\Delta_{s}^{(i)}=0$ non-zero solutions and, consequently, exact Padé approximants will be obtained for equation (18) in the given approximation in terms of $c$.

Hence the following is a necessary condition for convergence of a succession of Padé approximants (16), at $s \rightarrow \infty$, to fractional rational functions,

$$
P^{(i)}=\sum_{j=0}^{\infty} a_{j}^{(i)} c^{j} / \sum_{j=0}^{\infty} b_{j}^{(i)} c^{j}, \quad b_{0}^{(i)}=1:
$$

namely,

$$
\lim _{s \rightarrow \infty} \Delta_{s}^{(i)}=0, \quad i=2,3, \ldots, n .
$$

Indeed, if the conditions (21) are not satisfied, non-zero values of the coefficients $a_{i}^{(i)}$ and $b_{i}^{(i)}$ in equation (20) will obviously not be obtained.

In addition, the limiting Padé approximants $P^{(i)}$ will be suited for the description of a solution at any value of amplitude $c$ if the functions $P^{(i)}$ have no poles.

Conditions (21) are necessary but not sufficient for the convergence of approximants (16) to functions (20); nevertheless, the role of conditions (21) is determined by the following considerations.

Since, in the general case, there are more than one quasi-linear local expansion (13) and essentially non-linear local expansions (14) alike, the numbers of expansions of the respective type being not necessarily equal, it is the convergence conditions (21) that allow one to establish a relation between the quasi-linear and essentially non-linear expansions: that is, to decide which of them corresponds to the same solution and which to different ones.

\section{EXAMPLES}

\subsection{EXAMPLE 1}

As a first example of an analysis based on the above technique, consider a conservative system with two degrees of freedom, the potential energy of which contains terms of the second and fourth powers of the variables $z_{1}$ and $z_{2}$. Writing $z_{1}=c x$ and $z_{2}=c y$, where $c=z_{1}(0)(x(0)=1)$, one obtains

$$
\begin{aligned}
& V=c^{2}\left(d_{1} \frac{x^{2}}{2}+d_{2} \frac{y^{2}}{2}+d_{3} x y\right)+c^{4}\left(\gamma_{1} \frac{x^{4}}{4}+\gamma_{2} x^{3} y+\gamma_{3} \frac{x^{2} y^{2}}{2}+\gamma_{4} x y^{3}+\gamma_{5} \frac{y^{4}}{4}\right) \\
& \equiv c^{2} V^{(2)}+c^{4} V^{(4)} .
\end{aligned}
$$

The equation for determining the trajectory $y(x)$ is of the form

$$
2 y^{\prime \prime}(h-V)+\left(1+y^{\prime 2}\right)\left(-y^{\prime} V_{x}+V_{y}\right)=0,
$$

while the boundary conditions (13) can be written as

$$
\left.\left(-y^{\prime} V_{x}+V_{y}\right)\right|_{h-v}=0 .
$$

On the surface $h-V=0$ (at $\dot{x}=\dot{y}=0$ ) one of the values of the variable $x$ is $x(0)=1$. The corresponding boundary condition is

$$
\left.\left(-y^{\prime} V_{x}+V_{y}\right)\right|_{x=1}=0 .
$$

In view of the system symmetry with respect to the origin of co-ordinates, the second boundary condition (at $x=-1$ ) coincides with condition (23). 
Inasmuch as the trajectory will be represented in terms of $c^{2}$ or $c^{-2}$ power series, introduce a parameter $v\left(v=c^{2}\right.$ in the quasi-linear case and $v=c^{-2}$ in the essentially non-linear case). Now the solutions of equation (22) can be found in the form of a series in terms of the small parameter $v$ :

$$
y=\sum_{j=0}^{\infty} y_{j}(x) v^{j}
$$

In equation (22) and in the boundary conditions (23), $V=V_{0}+v V_{1}$ and $h=h_{0}+v h_{1}$; also, in the quasi-linear case, $v=c^{2}, V_{0}=V^{(2)}, V_{1}=V^{(4)}, h_{0}=\left.V^{(2)}\right|_{x=1}$ and $h_{1}=\left.V^{(4)}\right|_{x=1}$; and in the essentially non-linear case, $v=c^{-2}, V_{0}=V^{(4)}, V_{1}=V^{(2)}, h_{0}=\left.V^{(4)}\right|_{x=1}$ and $h_{1}=\left.V^{(2)}\right|_{x=1}$.

In the zeroth approximation in $v$, both the linear and the non-linear homogeneous systems admit normal vibrations in the form $y=k_{0} x$. The constants $k_{0}$ are determined from an algebraic second (linear system) or fourth (non-linear system) degree equation which is obtained from equation (22):

$$
-k_{0} V_{0 x}\left(1, k_{0}\right)+V_{0 y}\left(1, k_{0}\right)=0 .
$$

For definiteness, let $d_{1}=d_{2}=1+\gamma, d_{3}=-\gamma, \gamma_{1}=1, \gamma_{2}=0, \gamma_{3}=3, \gamma_{4}=0 \cdot 2091$ and $\gamma_{5}=\gamma$. Write the equations of motion for such a system:

$$
\begin{gathered}
\ddot{x}+x+\gamma(x-y)+c^{2}\left(x^{3}+3 x y^{2}+0 \cdot 2091 y^{3}\right)=0, \\
\ddot{y}+y+\gamma(y-x)+c^{2}\left(2 y^{3}+3 x^{2} y+0 \cdot 6273 y^{2} x\right)=0 .
\end{gathered}
$$

In the linear limiting case $(c=0)$ two rectilinear normal modes of vibrations, $y=k_{0} x$, $k_{0}^{(1)}=1$ and $k_{0}^{(2)}=-1$, are obtained by using equations (25), while a non-linear system (equations of motion contain only third power terms with respect to $x$ and $y$ ) admits four such modes; $k_{0}^{(3)}=1.496, k_{0}^{(4)}=0, k_{0}^{(5)}=-1 \cdot 279$ and $k_{0}^{(6)}=-5$.

In order to determine nearly rectilinear curvilinear trajectories of normal vibrations, equation (22) is used along with the boundary conditions (23). In particular, the first approximation in $v$ gives the following expression for the trajectory:

$$
2 y_{1}^{\prime \prime}\left(h_{0}-V_{0}\right)+\left(1+k^{2}\right)\left[-y_{1}^{\prime} V_{0 x}+y_{1}\left(V_{0 y y}-k V_{0 x y}\right)-k V_{1 x}+V_{1 y}\right]=0 .
$$

Here, $y=k x$ is substituted in all functions $V_{0}$ and $V_{1}$ and their derivatives. Equations in the subsequent approximations in terms of $v$ are obtained similarly. Such splitting by a small parameter should be performed in the boundary conditions (23) as well.

As mentioned above, the solution is sought in the form of a power series in terms of $v$ and $x\left(v=c^{2}\right)$ in the quasi-linear case and $v=c^{-2}$ in the essentially non-linear case). One finds (the clumsy intermediate calculations are omitted) the following relationships for $\rho=\rho(v)$ for various values of $\gamma$ and all types of vibrations (terms of the order $v^{2}$ and $x^{3}$ were retained):

$$
\begin{array}{cc}
\gamma=2 & \gamma=0 \cdot 5 \\
\text { (quasi-linear case, } \quad v=c^{2} \text { ) } & \gamma=0 \cdot 2 \\
\text { I } \rho_{1}^{(1)}=1-0 \cdot 177 v-0 \cdot 435 v^{2} & \rho_{1}^{(1)}=1-2 \cdot 6125 v-4 \cdot 721 v^{2} \\
\text { II } \rho_{2}^{(1)}=-1-0 \cdot 112 v+0 \cdot 050 v^{2} & \rho_{2}^{(1)}=1-1 \cdot 013 v-1 \cdot 140 v^{2} \\
\rho_{2}^{(1)}=-1-0 \cdot 445 v+0 \cdot 686 v^{2}
\end{array}
$$

(essentially non-linear case, $\quad v=c^{-2}$ ) 


$$
\rho_{3}^{(2)}=1 \cdot 496+0 \cdot 183 v+0 \cdot 029 v^{2}
$$

$\mathrm{IV} \rho_{4}^{(2)}=1.667 v-2 \cdot 871 v^{2}$

$$
\rho_{4}^{(2)}=0 \cdot 133 v+0 \cdot 036 v^{2}
$$

$$
\rho_{4}^{(2)}=0 \cdot 333 v+0 \cdot 098 v^{2}
$$

$$
\begin{gathered}
\mathrm{V} \rho_{5}^{(2)}=-1 \cdot 279+0 \cdot 767 v-2 \cdot 273 v^{2} \\
\rho_{5}^{(2)}=-1.279+0 \cdot 192 v-0 \cdot 158 v^{2}
\end{gathered}
$$

VI $\rho_{6}^{(2)}=-5-3 \cdot 165 v+1 \cdot 863 v^{2}$

$$
\rho_{6}^{(2)}=-5-0 \cdot 3165 v+0 \cdot 113 v^{2}
$$

$$
\rho_{6}^{(2)}=-5-0 \cdot 791 v+0.696 v^{2} .
$$

Matching with the use of Padé approximants of the type (20) shows that only for the pairs of expansions (I), (IV) and (II), (V) is an increase of the number $s$ accompanied by a decrease in the determinant $\Delta_{s}$; i.e., condition (21) is satisfied. Therefore, each of the couples corresponds to a periodic solution.

By matching of the local expansions the following Padé approximants ( $\delta$ is an error of coefficient computation) are obtained:

$$
\begin{aligned}
& \gamma=2 \\
& \gamma=0 \cdot 5 \\
& \gamma=0 \cdot 2 \\
& \text { I-IV } \quad \rho=\frac{1+1 \cdot 20 c^{2}}{1+1 \cdot 61 c^{2}+0 \cdot 72 c^{4}} \\
& (\delta \simeq 8 \%) \\
& \rho=\frac{1+1 \cdot 70 c^{2}}{1+3 \cdot 96 c^{2}+13 \cdot 29 c^{4}} \\
& (\delta \simeq 13 \%) \\
& \rho=\frac{1+1 \cdot 06 c^{2}}{1+2 \cdot 06 c^{2}+3 \cdot 20 c^{4}} \\
& (\delta \simeq 1 \%) \\
& \text { II-V } \quad \rho=\frac{-1-1 \cdot 11 c^{2}-0 \cdot 275 c^{4}}{1+1 \cdot 00 c^{2}+0 \cdot 215 c^{4}} \\
& (\delta \simeq 4 \%) \\
& \rho=\frac{-1-6 \cdot 41 c^{2}-9 \cdot 03 c^{4}}{1+5 \cdot 30 c^{2}+7 \cdot 02 c^{4}} \\
& (\delta \simeq 1 \%) \\
& \rho=\frac{-1-2 \cdot 76 c^{2}-1 \cdot 36 c^{4}}{1+2 \cdot 31 c^{2}+1 \cdot 04 c^{4}} \\
& (\delta \simeq 4 \%)
\end{aligned}
$$

The two additional modes of vibration, expansions (III) and (VI), exist only in a non-linear system; as $v$ increases (amplitude $c$ decreases), they vanish at a certain limiting point.

For the analysis of these vibration modes, assume a new variable $\sigma=(\rho-1 \cdot 496) /$ $(\rho+5)$. Represent $v$ in terms of a series of powers of $\sigma$ (expansion (III)) or $\sigma^{-1}$ (expansion (VI)). Restricting oneself to terms with a power less than or equal to three, one obtains the following:

$$
\gamma=2 \quad \gamma=0 \cdot 5 \quad \gamma=0 \cdot 2
$$

III $v=8 \cdot 874 \sigma-67 \cdot 512 \sigma^{2}+1171 \cdot 110 \sigma^{3}$

$$
\begin{aligned}
v=35 \cdot 497 \sigma-164 \cdot 184 \sigma^{2}+1882 \cdot 648 \sigma^{3} \\
v \\
v=88 \cdot 986 \sigma-197 \cdot 460 \sigma^{2}+570 \cdot 389 \sigma^{3}
\end{aligned}
$$


IV $v-2 \cdot 052 \sigma^{-1}-5 \cdot 566 \sigma^{-2}+21 \cdot 111 \sigma^{-3}$

$$
\begin{aligned}
v=8 \cdot 212 \sigma-67 \cdot 556 \sigma^{-1} & +982 \cdot 913 \sigma^{-2} \\
v & =20 \cdot 492 \sigma^{-4}+170 \cdot 182 \sigma^{-2}+2506 \cdot 770 \sigma^{-3} .
\end{aligned}
$$

By using the variable $\sigma$ two expansions in terms of positive and negative powers have been obtained; therefore, fractional rational representations can be introduced as above. By comparing these expansions, the following Padé approximants are obtained:

$$
\begin{array}{ccc}
\gamma=2 & \gamma=0 \cdot 5 & \gamma=0 \cdot 2 \\
v=\frac{8 \cdot 874 \sigma+1 \cdot 126 \sigma^{2}}{1+4 \cdot 300 \sigma+2 \cdot 836 \sigma^{2}+0 \cdot 549 \sigma^{3}} & \\
v=\frac{35 \cdot 497 \sigma+5 \cdot 108 \sigma^{2}}{1+3 \cdot 021 \sigma-0 \cdot 794 \sigma^{2}+0 \cdot 622 \sigma^{3}} \\
v=\frac{88 \cdot 986 \sigma+1 \cdot 470 \sigma^{2}}{1-0 \cdot 143 \sigma+3 \cdot 747 \sigma^{2}+0 \cdot 072 \sigma^{3}} .
\end{array}
$$

Now produced to the determination of the limiting point. Obviously, it can be found from $\partial v / \partial \sigma=0$. From equation (29), at $\gamma=2$ the limiting point is $v \simeq 1 \cdot 21, c \simeq 0.91$, at $\gamma=0.5$ it is $v \simeq 11 \cdot 10, c \simeq 0.30$, and at $\gamma=0.2$ it is $v \simeq 23.93, c \simeq 0.20$. Hence, as $\gamma \rightarrow 0$ the limiting point is characterized by the amplitude $c \rightarrow 0$. Therefore, the two additional vibration modes in a non-linear system can exist at rather small amplitudes of vibration. Note that quasi-linear analysis does not allow one to find these solutions, even at small amplitudes.

In the limit, when $\gamma=0$, a linear system decomposes into two independent oscillators having identical frequencies and admits any rectilinear modes of normal vibrations. Obviously, a full system (25) at $\gamma=0$ admits four modes of vibrations (in a non-linear case) $y_{2}=k y_{1}, k=(1 \cdot 496,0,-1 \cdot 279,-5)$.

Thus fractional rational Padé approximants allow judgement of non-local behavior of normal vibrations in non-linear finite-dimensional systems. For systems (25) the evolution of modes of normal vibrations is shown in Figure 1 by using parameters $c$ and $\varphi=\operatorname{arctg} \rho$ (the figure shows periodicity in $\varphi$, the period being $2 \pi)$. The analytical solutions ((27) and (29) were employed) and numerical check computations (carried out by A. L. Zhupiev at $\gamma=2$ ) show good agreement. For solutions II-V, for instance, relationship (29) and the numerical calculation gave, in the scale selected, the same curve (see Figure 1).

Note that the systems considered above (systems with cubic non-linearity) can be obtained in calculations of non-linear vibrations of shells (when using the BubnovGalerkin technique) as well as in other problems. For instance, consider a problem of the vibrations of a load on resilient supports under a force having a constant direction (see Figure 2). Assume that the restoring forces on the left and right supports respectively are $F_{1}=d_{1} q_{1}+r_{1} q_{1}^{3}$ and $F_{2}=d_{2} q_{2}+r_{2} q_{2}^{3}$, where $q_{1}$ and $q_{2}$ are vertical displacements of the supports and $Q=d_{3} x_{1}+r_{3} x_{1}^{3}$ is the constantly directed force. Let $m$ be the load weight, and let $m \rho^{2}$ be the moment of inertia with respect to an axis going through the center of mass.

The equations of motion for the system are

$$
\begin{gathered}
m \ddot{x}_{1}+d_{1}\left(x_{1}+a x_{2}\right)+r_{1}\left(x_{1}+a x_{2}\right)^{3}+d_{2}\left(x_{1}-b x_{2}\right)+r_{2}\left(x_{1}-b x_{2}\right)^{3}+d_{3} x+r_{3} x^{3}=0, \\
\rho^{2} m \ddot{x}_{2}+d_{1}\left(x_{1}+a x_{2}\right) a+r_{1}\left(x_{1}+a x_{2}\right)^{3} a+d_{2}\left(x_{1}-b x_{2}\right) b-r_{2}\left(x_{1}-b x_{2}\right)^{3} b=0 .
\end{gathered}
$$




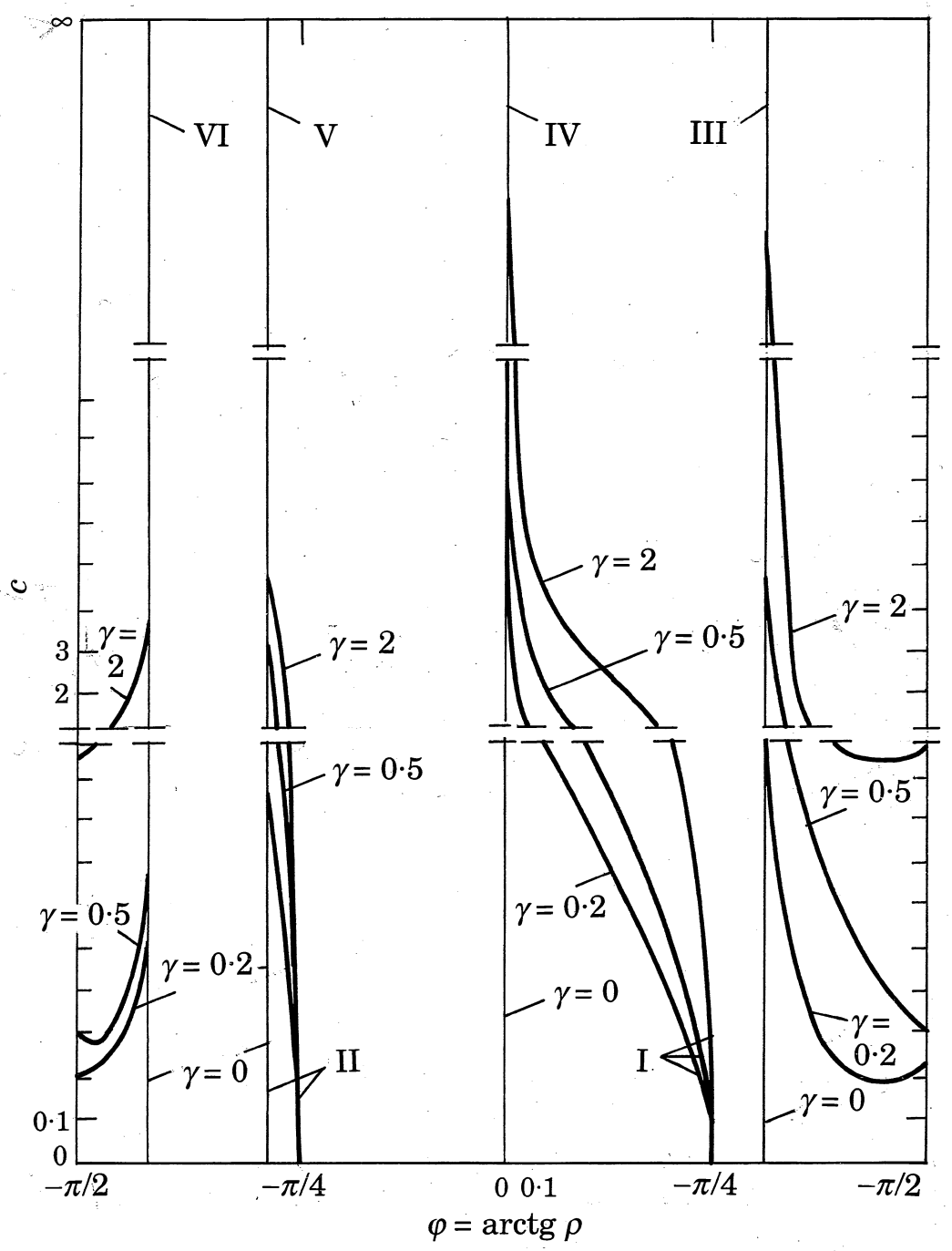

Figure 1. The evolution of normal vibration modes for system $(25)$ in parameters $c$ and $\varphi=\operatorname{arctg} \rho(c$ and $\rho$ are amplitudes of position co-ordinates). Local normal mode expansions are designated by I, II (quasi-linear case) and III, IV, V and VI (essentially non-linear case).

At certain numerical values of coefficients $m, \rho, a, b, d_{1}, r_{1}, d_{2}, r_{2}, d_{3}$ and $r_{3}$, equations of motion in the form of equations (25) are obtained. The behavior of the vibrations for system (25) is shown in Figure 1.

\subsection{EXAMPLE 2}

Johnson and Rand [8] gave results of numerical integration for a conservative system with potential energy

$$
V(x, y)=\frac{1}{2}\left(a \xi^{2}+b \eta^{2}\right)+\left(3 x^{4}+y^{4}\right)
$$

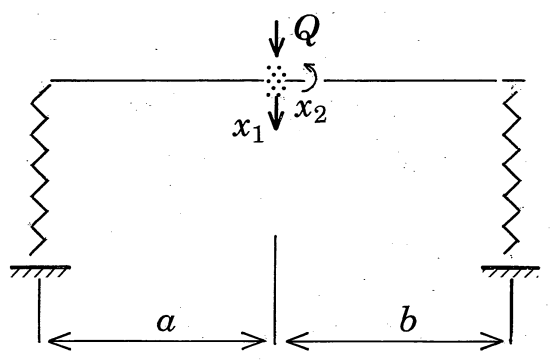

Figure 2. A load on resilient supports under a force having a constant direction (two-degree-of-freedom system). 
where $\xi=\xi(x, y)=x \cos \theta+y \sin \theta$ and $\eta \equiv \eta(x, y)=-x \sin \theta+y \cos \theta$, at various values of parameters $a, b, \theta$ and energy $h$. All calculations given here are classified into three cases: (1) $a=8, b=4, \theta=\pi / 4, h=40,70,100$; (2) $a=8, b=4, \theta=0, h=10,24$; (3) $a=32, b=2, \theta=\pi / 4, h=10$. Plots representing trajectories on a configurational plane $(x, y)$ show that, as $h$ increases, two normal vibration modes (two trajectories) are superseded by four: i.e., bifurcation takes place.

Calculations (with E. V. Ladygina) carried out using Padé approximants allow one to follow changes in the normal vibration modes as the energy increases from 0 to infinity (at a fixed value of $\theta$ ) and, importantly, to gain an insight into the evolution of the normal vibrations with the variation in parameter $\theta$.

The limiting linear system has two normal vibration modes $y=k^{(1)} x, k^{(1)}$ varying with $\theta$. The limiting non-linear homogeneous system, which is determined by the part of the potential energy containing fourth power terms, also admits solutions in the form $y=k^{(2)} x$, where in all cases $k^{(2)}=-\sqrt{3},+\sqrt{3}, 0, \infty$.

The results obtained are given below for a situation with $a=8$ and $b=4$. The evolution of normal vibration modes with vibration in energy (amplitude) from 0 to infinity and in $\theta$ is shown in Figure 3, where $c=x(0), \rho=y(0)$ and $\varphi=\operatorname{arctg} \rho$. Digits refer to the following cases: (1) $\theta=\pi / 4$; (2) $\theta=2 \pi / 9$; (3) $\theta=\pi / 6$; (4) $\theta=\pi / 9$; (5) $\theta=\pi / 18$; (6) $\theta=0$. The fractional rotational representations of the in-phase and out-of-phase vibration modes which exist in the system at any amplitude $c$, and are shown as (I) and (II) in Figure 3, are as follows:

(I)

$$
\begin{array}{lll}
\theta=\pi / 4 & \rho=\frac{1}{1+0 \cdot 999 c^{2}} & \rho=\frac{-1-1.997 c^{2}}{1+1 \cdot 153 c^{2}} \\
\theta=2 \pi / 9 & \rho=\frac{0 \cdot 839}{1+1 \cdot 127 c^{2}} & \rho=\frac{-1 \cdot 192-2 \cdot 155 c^{2}}{1+1 \cdot 244 c^{2}} \\
\theta=\pi / 6 & \rho=\frac{0 \cdot 577}{1+c^{2}} & \rho=-\sqrt{ } 3
\end{array}
$$

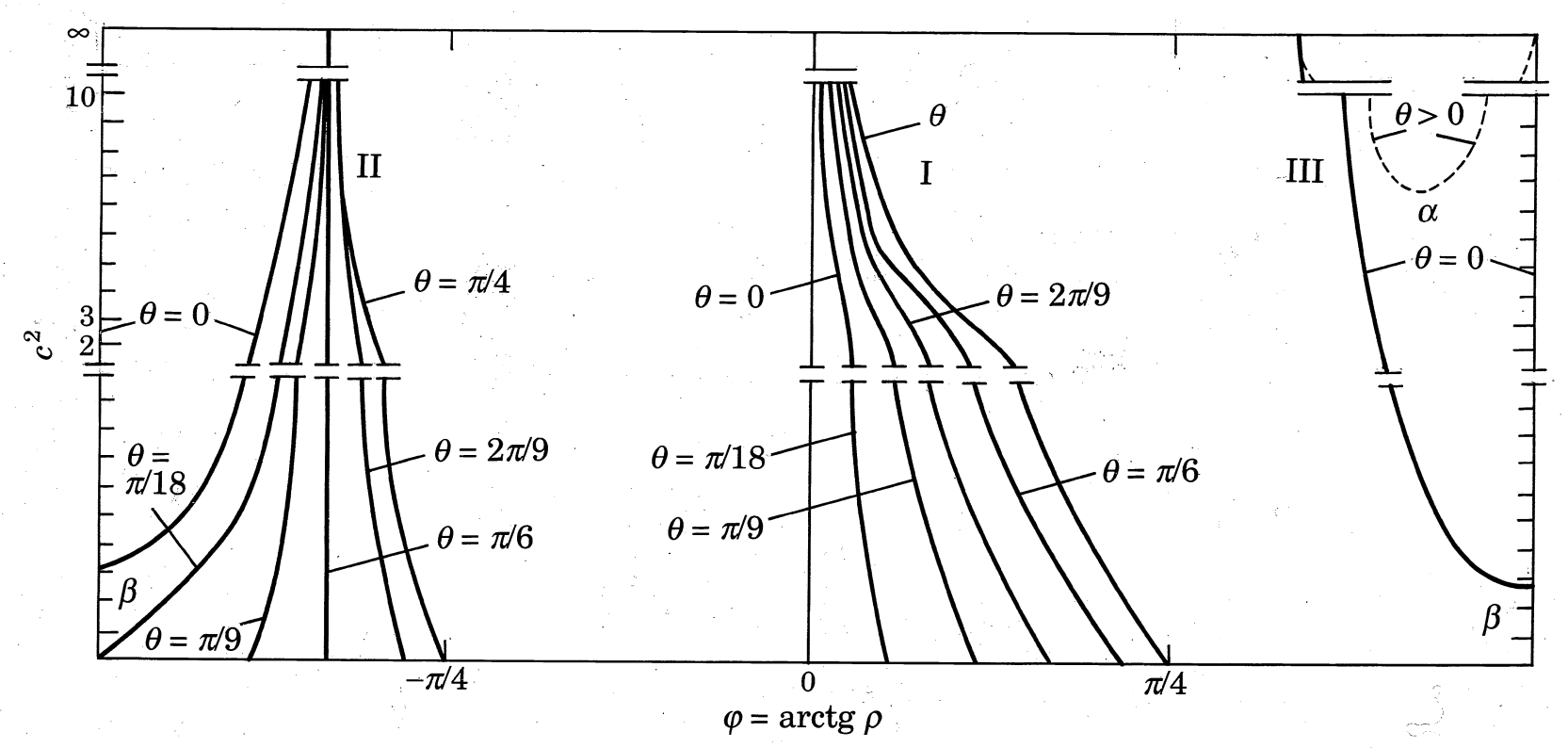

Figure 3. The evolution of normal vibration modes for system (30) in parameters $c$ and $\varphi=\operatorname{arctg} \rho$ ( $c$ and $\rho$ are amplitudes of position co-ordinates). Normal vibration modes are designated by I, II and III; $\alpha$ is the limiting point (at $\theta>0$ ), while $\beta$ is a bifurcation point (at $\theta=0$ ). 


$$
\begin{array}{lll}
\theta=\pi / 9 & \rho=\frac{0 \cdot 364}{1+0 \cdot 849 c^{2}} & \rho=\frac{-2 \cdot 747-4 \cdot 050 c^{2}}{1+2 \cdot 338 c^{2}} \\
\theta=\pi / 18 & \rho=\frac{0 \cdot 176}{1+0 \cdot 773 c^{2}} & \rho=\frac{-5 \cdot 671-8 \cdot 360 c^{2}}{1+4 \cdot 827 c^{2}} \\
\theta=0 & \rho=0 & \rho=\infty .
\end{array}
$$

(III) in Figure 3 refers to additional vibration modes (at $\theta=0$ ) which exist only at great amplitudes and that vanish at a certain point as the amplitude decreases.

The calculated results show that at $\theta>0$ vibration modes are changed and the limiting point ( $\alpha$ in the figure) is replaced by a bifurcation point $\beta$.

\section{REFERENCES}

1. R. M. Rosenberg 1962 Journal of Applied Mechanics 29, 7-14. The normal modes of nonlinear $n$-degree-of freedom systems.

2. R. M. Rosenberg 1966 Advances of Applied Mechanics 9, 156-243. New York: Academic Press. On nonlinear vibrations of systems with many degrees of freedom.

3. L. I. Manevich, Yu. V. Mikhlin and V. N. Pilipchuk 1989 The Method of Normal Oscillation for Essentially Nonlinear Systems. Moscow: Nauka.

4. A. M. Liapunov 1950 The General Problem of the Stability of Motion. Moscow: Gostehizdat (reprint of original thesis, 1892).

5. Yu. V. Mikhlin 1985 Applied Mathematics and Mechanics 49(5), 738-743. Joining of local expansions in the nonlinear oscillations theory.

6. G. A. Baker and P. Graves-Morris 1981 Padé Approximants. London: Addison-Wesley.

7. G. A. BAKer 1975 Essentials of Padé Approximants. New York: Academic Press.

8. T. L. JoHnSON and A. H. RAND 1979 International Journal of Non-linear Mechanics 14(1), 1-12. On the existence and bifurcation of minimal normal modes. 